\title{
A coherent transcriptional feed-forward motif model for mediating auxin-sensitive PIN3 expression during lateral root development
}

Qian Chen ${ }^{1,2,3}$, Yang Liu'1,2,4, Steven Maere ${ }^{1,2}$, Eunkyoung Lee ${ }^{5}$, Gert Van Isterdael ${ }^{1,2}$, Zidian Xie ${ }^{6}$, Wei Xuan ${ }^{1,2}$, Jessica Lucas ${ }^{5}$, Valya Vassileva ${ }^{1,2,7}$, Saeko Kitakura ${ }^{1,2,8}$, Peter Marhavý9,10, Krzysztof Wabnik ${ }^{1,2,9}$, Niko Geldner ${ }^{10}$, Eva Benková ${ }^{9}$, Jie Le ${ }^{11}$, Hidehiro Fukaki ${ }^{12}$, Erich Grotewold ${ }^{6}$, Chuanyou Li ${ }^{3}$, Jiři Friml ${ }^{1,2,9}$, Fred Sack ${ }^{5, \ddagger}$ Tom Beeckman ${ }^{1,2} \&$ Steffen Vanneste ${ }^{1,2}$

Multiple plant developmental processes, such as lateral root development, depend on auxin distribution patterns that are in part generated by the PIN-formed family of auxin-efflux transporters. Here we propose that AUXIN RESPONSE FACTOR7 (ARF7) and the ARF7-regulated FOUR LIPS/MYB124 (FLP) transcription factors jointly form a coherent feed-forward motif that mediates the auxin-responsive PIN3 transcription in planta to steer the early steps of lateral root formation. This regulatory mechanism might endow the PIN3 circuitry with a temporal 'memory' of auxin stimuli, potentially maintaining and enhancing the robustness of the auxin flux directionality during lateral root development. The cooperative action between canonical auxin signalling and other transcription factors might constitute a general mechanism by which transcriptional auxin-sensitivity can be regulated at a tissue-specific level.

\footnotetext{
${ }^{1}$ Department of Plant Systems Biology, VIB, Ghent 9052, Belgium. ${ }^{2}$ Department of Plant Biotechnology and Bioinformatics, Ghent University, Ghent 9052 , Belgium. ${ }^{3}$ State Key Laboratory of Plant Genomics, National Centre for Plant Gene Research, Institute of Genetics and Developmental Biology, Chinese Academy of Sciences, Beijing 100101, China. ${ }^{4}$ Biotechnology Research Institute, Chinese Academy of Agricultural Sciences, Beijing 100081, China.

${ }^{5}$ Department of Botany, University of British Columbia, Vancouver, British Columbia V6T 1Z4, Canada. ${ }^{6}$ Center for Applied Plant Sciences (CAPS) and Department of Molecular Genetics, The Ohio State University, Columbus, Ohio 43210, USA. ${ }^{7}$ Institute of Plant Physiology and Genetics, Bulgarian Academy of Sciences, Academik Georgi Bonchev Street, Sofia 1113, Bulgaria. ${ }^{8}$ Department of Biological Sciences, Graduate School of Science, Osaka University, Osaka 560-0043 Japan. ${ }^{9}$ Institute of Science and Technology Austria, Klosterneuburg 3400, Austria. ${ }^{10}$ Department of Plant Molecular Biology, UNIL-Sorge, University of Lausanne, Lausanne 1015, Switzerland. ${ }^{11}$ Key Laboratory of Plant Molecular Physiology, Institute of Botany, Chinese Academy of Sciences, Beijing 100093, China. ${ }^{12}$ Department of Biology, Graduate School of Science, Kobe University, 1-1 Rokkodai, Kobe 657-8501, Japan. Correspondence and requests for materials should be addressed to T.B. (email: tobee@psb.vib-ugent.be) or to S.V. (email: stnes@psb.vib-ugent.be).

$\ddagger$ Deceased on June 30, 2015.
} 
P lant growth involves the reiterative formation of postembryonic organs such as lateral roots (LRs), tailoring the root architecture to environmentally imposed limitations. Typically, LRs are regularly spaced along the main root. The mechanism that controls this regular spacing involves oscillatory gene expression in the elongation zone of the primary root meristem $^{1}$, also called the root clock $^{2}$. This mechanism defines groups of cells that are competent to form LR primordia (prebranch sites). Xylem pole pericycle cells within such prebranch sites can become LR founder cells (FCs) that subsequently can be activated to initiate a LR primordium ${ }^{3}$. Throughout subsequent developmental stages, the nascent LR primordium communicates with the overlaying root tissues to facilitate LR emergence ${ }^{4-7}$.

The plant hormone auxin commonly plays a master role in organogenesis and morphogenesis ${ }^{8}$, as is explicit for LR development ${ }^{9}$. In the lateral root cap, the auxin precursor indole-3-butyric acid is converted to indole-3-acetic acid (IAA), feeding auxin into the root clock, thereby stimulating prebranch site formation ${ }^{10}$. Auxin accumulation drives the acquisition of FC identity in pericycle cells ${ }^{11}$, and the subsequent transition from FC to LR initiation requires stabilization of auxin signalling in these cells ${ }^{12}$. Subsequent LR patterning and morphogenesis are orchestrated by dynamic auxin flows ${ }^{6,13,14}$ as well as by complex interactions with surrounding tissues ${ }^{4,5,7,15}$.

Auxin transporters of the PIN-formed (PIN) family ${ }^{16}$ contribute to nearly every step of these regulatory auxin flows $6,12,13$. Prominently among the LR-regulating PINs is PIN3, which is transiently expressed in the endodermis cells overlaying FCs to promote their transition to LR initiation ${ }^{12}$, and is later in development also expressed in LR primordiumoverlaying tissues to facilitate LR emergence ${ }^{6}$. These examples demonstrate the importance of dynamic PIN expression patterns in LR development.

The canonical auxin signalling pathway has been implicated as the main mechanism for regulating PIN expression ${ }^{6,17}$. This pathway is defined by the auxin-induced proteolysis of transcriptional repressor of the Aux/IAA family, thereby derepressing auxin response transcription factors $(\mathrm{ARFs})^{8}$. Expression of different combinations of members of both protein families within a single cell is believed to define different auxin responsive transcriptional outputs ${ }^{18-20}$. Detailed analyses of dynamic transcriptional changes associated with auxin-induced and gravity-induced LR formation start revealing the underlying gene regulatory networks ${ }^{21}$. Besides the canonical auxin signalling pathway, several other transcription factors have been implicated in transcriptional control over PINs; the MADSbox XAANTAL2 (XAL2/AGL14) and INDETERMINATE DOMAIN (IDD) (IDD14, IDD15, IDD16) transcription factors were recently identified as direct regulators of PIN1 and/or PIN4 through direct binding to the respective promoters ${ }^{22,23}$. However, almost nothing is known about how such regulators bind to cis-elements in the PIN regulatory regions and how they are integrated with the canonical auxin signalling pathway to regulate PIN expression during organogenetic processes such as LR development.

We previously demonstrated that PIN3 shows remarkably dynamic expression during the patterning of stomatal complexes in the leaf epidermis ${ }^{24}$. We thus investigated whether the mechanisms controlling PIN3 expression are similar during stomatal and LR development. Two genes involved in the late steps of stomatal development encode the transcription factors FOUR LIPS (FLP)/MYB124 and its closest paralogue MYB88 (ref. 25). FLP and MYB88 act redundantly to restrict guard mother cell proliferation, in part by repressing the expression of cell cycle genes ${ }^{26-28}$. Here, we propose that FLP transcription is auxin responsive, downstream of ARF7, and that FLP binds directly to the PIN3 promoter as part of the molecular mechanism that controls its auxin-sensitive expression. Together, FLP and ARF7 could define a coherent feed-forward motif that controls PIN3 expression. Mathematical modelling reveals that such transcriptional circuit could generate a temporal 'memory' of auxin stimuli, potentially enhancing the robustness of the auxin flux patterns. Consistently, the corresponding cisregulatory modules in the $P I N 3$ promoter are required for normal auxin-responsive PIN3-YFP expression that can complement the LR defect in pin3 mutants. The feed-forward transcriptional circuit that we propose for auxin-sensitive PIN3 transcription thus seems critical for PIN3-controlled LR development.

\section{Results}

FOUR LIPS is a direct target of ARF7. While FLP is best known for its role in the late stages of stomatal development ${ }^{25}$, we observed prominent FLP promoter activity in developing LR primordia (Fig. 1a; Supplementary Fig. 1a). Given the importance of auxin in triggering LR development, we probed whether FLP expression is induced by auxin. In auxin-treated roots, transcripts of PIN3, as well as the expression of GATA23 and ACR4, which are hallmarks of LR initiation ${ }^{29,30}$, were rapidly upregulated (Fig. 1b). Similarly, transcripts of FLP, but not of MYB88, were also strongly auxin responsive (Fig. 1b). This auxin-activated FLP expression can be found in xylem pole pericycle cells, the cell type where LRs originate from (Supplementary Fig. 1b). The auxin signalling pathway that is central to LR induction depends largely upon the presence of the Auxin Response transcription Factor7 (ARF7) and SOLITARY ROOT/IAA14 (SLR) ${ }^{9}$. Together, these proteins define a canonical auxin pathway that regulates transcriptional changes associated with auxin-induced LR initiation. High auxin levels activate ARF7 transcriptional activity by destabilizing its interaction partner SLR/IAA14 (ref. 31). A gain-of-function mutation in SLR/IAA14 (slr-1), or loss-offunction mutations in the $A R F 7$ and $A R F 19$ genes disrupt auxin signalling to the extent that the respective (primary) roots are completely devoid of $\operatorname{LRs}^{32,33}$. We then tested whether the auxin inducible expression of FLP depends upon SLR/IAA14-ARF7based auxin signalling. In $s l r-1$ and in proARF7::ARF7-GR/arf7 arf19 mutants, FLP expression was reduced and almost completely insensitive to auxin treatment (Fig. 1c). Moreover, when ARF7 functionality was restored to proARF7::ARF7-GR/arf7 arf19 via dexamethasone (DEX/NAA co-treatment), FLP transcription regained auxin-responsiveness. A nearly identical expression profile was found for PIN3 (Fig. 1d), which suggests it represents a direct target of this auxin signalling pathway, 17. FLP contains two canonical AuxREs in the $5^{\prime}$ region upstream of its start codon (included in FLP_P2 amplicon) (Fig. 1e). Together with its early, SLR/IAA14-ARF7-dependent expression, we hypothesized that FLP could be a direct target of ARF7. Therefore, we performed ChIP analyses using anti-GR antibodies on proARF7::ARF7-GR/ $\operatorname{arf7}$ arf19. In the absence of DEX, none of the fragments were significantly enriched (Fig. 1f). By contrast, the FLP_P2 amplicon became significantly enriched when plants were treated with DEX, while the enrichment of neither FLP_P1 nor ACTIN2 changed significantly (Fig. 1f). Together these data demonstrate that FLP is a direct target of ARF7.

A role for FLP and MYB88 in PIN3-regulated LR development. To evaluate the relative contributions of FLP and MYB88 expression to LR development, we analysed the LR phenotypes of corresponding single and double mutants (Fig. 2a; Supplementary Fig. 2a,b). Although myb88 single mutants showed a wild-type (WT) root phenotype, the $f l p-1$ and $f l p-7$ alleles each exhibited reduced numbers of LRs, including a reduction in the earliest 
a
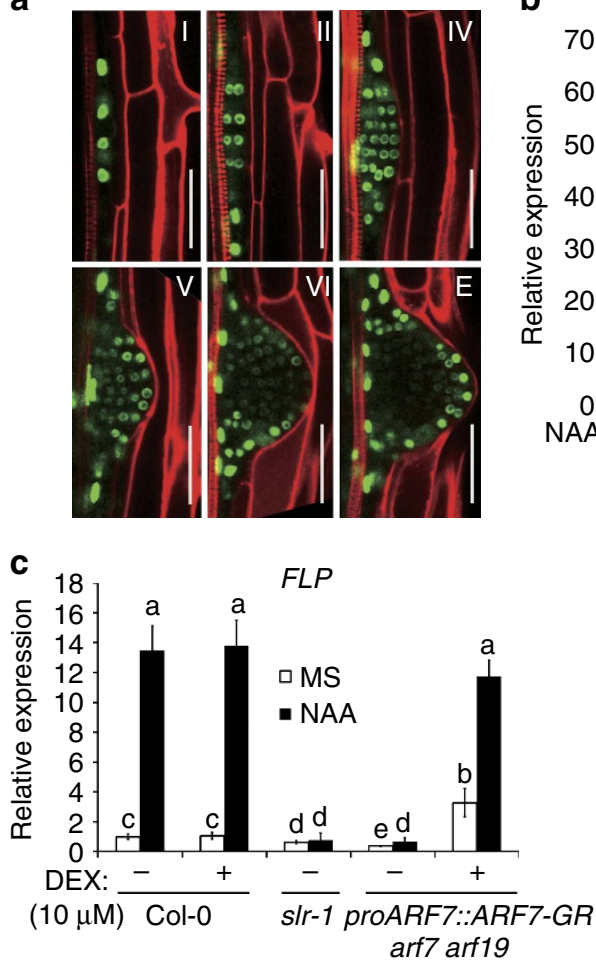

e FLP promoter b

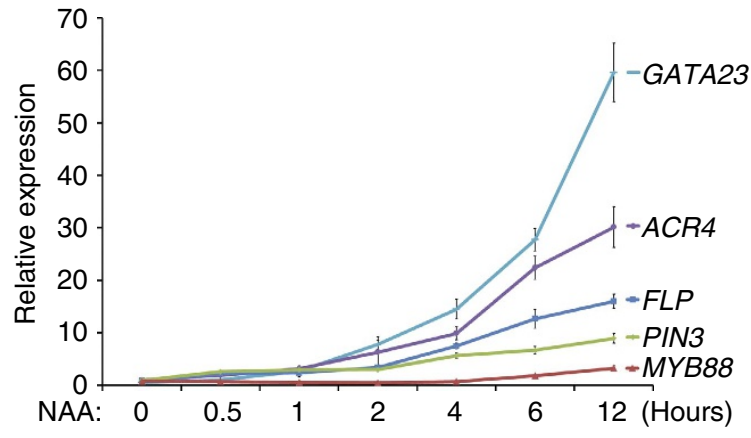

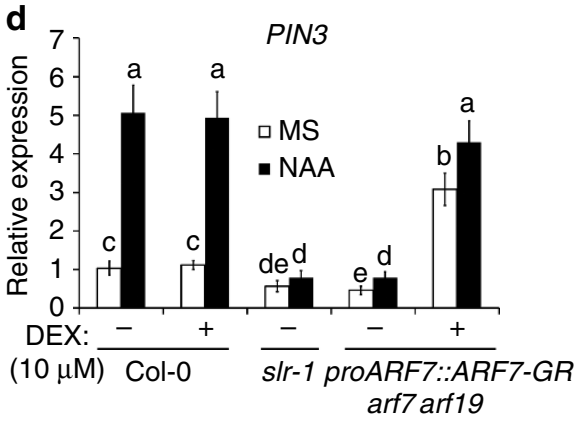

f

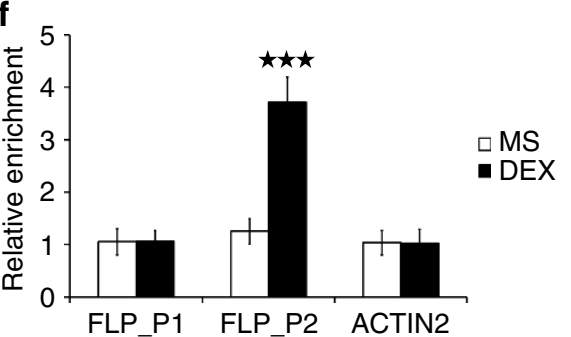

Figure 1 | FLP is a direct target of ARF7. (a) proFLP::NLS-GFP expression in different stages of LR development. Scale bar, $50 \mu \mathrm{m}$. (b) qRT-PCR analysis of GATA23, ACR4, PIN3, FLP and MYB88 expression during a $12 \mathrm{~h}$ auxin (10 $\mu \mathrm{M}$ NAA) time course from roots of 6-day-old WT seedlings. Expression levels were normalized to those of ACTIN2. (c,d) Relative auxin-inducibility ( $6 \mathrm{~h}, 10 \mu \mathrm{M} \mathrm{NAA}$ ) of (c) FLP and (d) PIN3 in WT ( $\pm 10 \mu \mathrm{M}$ DEX for $6 \mathrm{~h}$ ), s/r-1, and proARF7::ARF7-GR/arf7 arf19 ( $\pm 10 \mu \mathrm{M}$ DEX for $6 \mathrm{~h}$ ). Samples with different letters are significantly different: $P<0.05$ (Fisher's LSD mean separation test). (e) Schematic presentation of the FLP promoter (3.0 kb upstream of the translational start site at position $(+1)$ with indication of the regions targeted for ChIP analysis (FLP_P1 and FLP_P2), and the presence of auxin response elements (AuxREs, black triangles) (f) Enrichment of the indicated DNA fragments (FLP_P1, and FLP_P2) following ChIP using anti-GR antibodies with proARF7::ARF7-GR/arf7 arf19 treated for $6 \mathrm{~h}$ with or without DEX. A fragment from the ACTIN2 promoter was tested as a negative control. $n=3$. Data are means $\pm \mathrm{s}$.d. ${ }^{\star \star \star} P<0.001$ (Student's $t$-test).

developmental stages. These phenotypes were more pronounced in the $f l p-1$ myb88 and in the $f l p-7$ myb88 double mutants. Our results thus reveal a key role for FLP in LR initiation, with MYB88 playing only a minor role, findings that echo the absence of obvious stomatal defects in the single myb88 mutants ${ }^{25}$, and the lack of persistent MYB88 expression in LR primordia (Supplementary Fig. 1c).

Given FLPs function in restricting proliferation in the stomatal cell lineage, and its necessity for cell proliferation during early LR development, we analysed the genetic interaction between $f l p-7$ myb88, and a triple mutant in A2-type cyclins (cyca2;234) at the level of LR formation (Supplementary Fig. 3). Consistent with the inherent proliferation requirement for LR formation, the cell proliferation-impaired cyca2;234 mutants ${ }^{26}$ exhibited a lower LR density than the WT, mostly resulting from a reduced level of LR initiation, a defect comparable to that of $f l p-7$ myb88. Strikingly, the quintuple mutant $f l p-7$ myb88 cyca2;234 developed much less LR initiation sites compared to cyca2;234 or flp-7 myb88 alone, consistent with LR initiation defects in cyca2;234 and flp-7 myb88 arising via distinct pathways, suggesting that the flp-7 myb88 LR defect is at least in part independent of the misregulated proliferation seen in cyca2;234.

Next, we addressed whether perturbation of LR development resulted from impaired auxin signalling. Therefore, we introgressed the synthetic auxin-response output reporter proDR5:: GUS into $f l p-7$ myb88 (Fig. 2b). Whereas normally this reporter is prominently expressed in developing LRs, the proDR5::GUS signal was only weakly detectable in LR primordia of $f l p-7$ myb88 double mutants (Fig. 2b). Because this reduced proDR5::GUS signal might have resulted from altered auxin signalling and/or distribution, we analysed the auxin responsiveness of proDR5:: GUS in WT and $f l p-7 m y b 88$. A $6 \mathrm{~h}$ auxin treatment equalized the GUS expression in both the WT and in $f l p-7$ myb88 (Fig. 2b). Moreover, both genotypes showed near-identical auxininduced increases of IAA19, GATA23 and ACR4 transcripts (Supplementary Fig. 4). These results argue against the idea that 

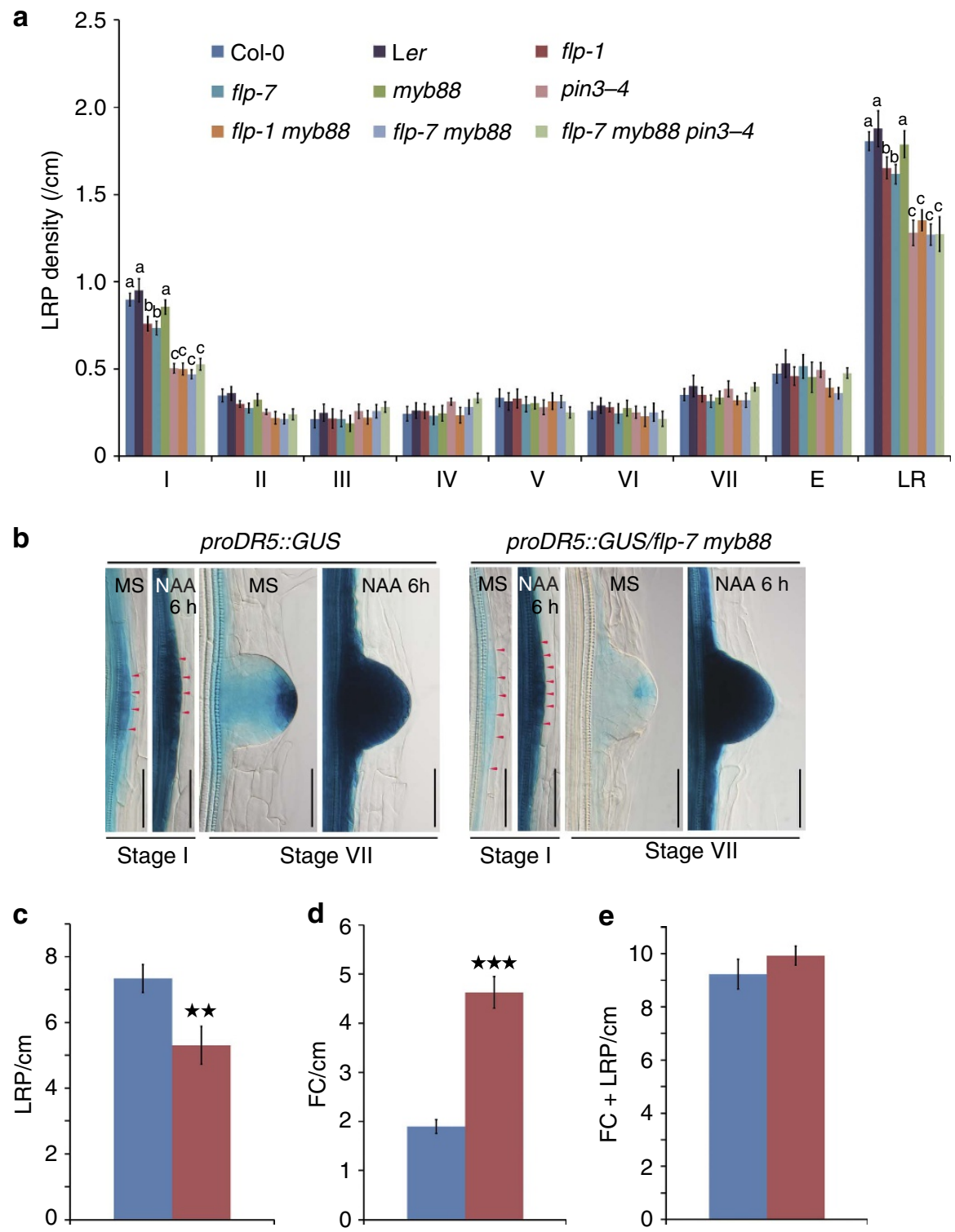

proDR5::GFP / WT

proDR5::GFP / flp-1 myb88

Figure 2 | Lateral root phenotypic analysis. (a) Densities of the different LR stages in 6-day-old seedlings of Col-0, Ler, flp-1, flp-7, myb88, pin3-4, flp-1 myb88, flp-7 myb88 and flp-7 myb88 pin3-4. $E=$ just emerged, not yet mature LRs; LR = mature LRs. Data shown are means \pm s.d. of at least three independent experiments, each time sampling $(n \geq 20)$. Samples in each stage with different letters are significantly different: $P<0.05$ (Fisher's LSD mean separation test). (b) Expression pattern of the auxin response output reporter proDR5::GUS in different LR stages of WT and flp-7 myb88 ( $\pm 10 \mu \mathrm{M}$ NAA for $6 \mathrm{~h}$ ). Scale bar, $50 \mu \mathrm{m}$. (c-e) Densities of (c) initiated LR primordia, (d) FCs and (e) the sum of FCs and initiated LR primordia in WT and flp-1 myb88 as visualized by proDR5::GFP. Data shown are means \pm s.e.m. and are representative of at least three independent experiments. (For each experiment, $n=10$ ). ${ }^{\star \star} P<0.01$ and ${ }^{\star \star \star} P<0.001$ (Student's $t$-test).

the LR defect in $f l p$ myb88 double mutants is due to a general reduction in auxin sensitivity. Instead, the altered proDR5::GUS expression level might be the result of abnormal auxin distribution in $f l p$ myb88 double mutants.

To further dissect the contribution of defects in auxin transport to the LR phenotype in $f l p m y b 88$, we focussed on the earliest visible stages of LR formation; namely LR FCs (undivided pericycle cells that exhibit proDR5::GFP expression) ${ }^{11}$ and LR initiation sites (FC that have undergone asymmetric cell division). Consistent with our other analyses (Fig. 2a), the densities of initiated LRPs were reduced in $f l p-1$ myb88 compared with the WT (Fig. 2c). In contrast, the FC densities in flp-1 myb88 had dramatically increased compared to WT (Fig. 2d), resulting in cumulated densities of FCs and initiated LRPs together that were comparable to WT (Fig. 2e). An identical LR defect with less initiated LRPs but more FCs was recently also described for pin3-4 (ref. 12). This striking resemblance prompted us to test for genetic interactions between $f l p-7$ myb88 and pin3-4. Analysis of different stages of LR development confirmed that pin3-4 and flp-7 myb88 LR defects were highly similar, with the most prominent defects appearing during initiation (Fig. 2a; Supplementary Fig. 2). Importantly, the LR phenotype in the flp-7 myb88 pin3-4 triple mutants showed near-identical reductions in LR initiation as the parental mutants. The absence of clear additive effects suggests that FLP, MYB88 and PIN3 act collectively in regulating LR initiation. 
PIN3 expression depends on FLP. Since the expression of both FLP and PIN3 are strongly auxin-inducible (Fig. 1b) and downstream of a common auxin signalling module (Fig. 1c,d), we tested whether auxin-induced PIN3 expression requires functional FLP protein. Indeed, proPIN3::PIN3-GFP showed reduced auxin-inducible expression in LR primordia when introgressed into $f p-1$ myb88 (Fig. 3a,b). In addition, we analysed PIN3 expression in proFLP::FLP-GR/flp-1 myb88 lines in which the LR defect, but not the stomatal phenotype, could be completely complemented by DEX-treatment (Supplementary Fig. 5). In this line, PIN1 expression levels exhibited auxin responsive amplitudes that were comparable to those of the WT, independently of DEX treatment (Fig. 3c). By contrast, in the absence of DEX, the auxin inducibility of PIN3 in roots was severely reduced (Fig. 3d). Importantly, DEX-treatment restored the auxin responsiveness of PIN3 to WT levels in a proFLP::FLP-GR/flp-1 myb88 background (Fig. 3d). These results show that FLP is required for auxin-induced PIN3 expression, as was also shown for PIN3 expression in the columella of the primary root meristem $^{34}$.

PIN3 is directly regulated by FLP and ARF7. Because both the expression level of FLP and PIN3 are relatively rapidly induced by auxin and depend upon SLR/IAA14-ARF7 signalling, we explored whether FLP might be a downstream effector of the auxin signalling pathway by directly regulating PIN3 expression.
Five different promoter fragments of the PIN3 promoter (FR1-5) were tested for their ability to interact with FLP via yeast-onehybrid assays (Fig. 4a). Only the fragment FR2 was found to generate Aureobasidin A (AbA)-resistant yeast growth, suggesting that this fragment can recruit FLP (Fig. 4b). This fragment contains two sites (FBS1 = AGCCG, FBS2 = TACCC) that meet the experimentally determined $[\mathrm{A} / \mathrm{T} / \mathrm{G}][\mathrm{A} / \mathrm{T} / \mathrm{G}] \mathrm{C}[\mathrm{C} / \mathrm{G}][\mathrm{C} / \mathrm{G}]$ consensus sequence for FLP binding ${ }^{27}$. Using short fragments containing either FBS, only the construct containing FBS1 could render the yeast resistant to AbA (Fig. 4b). Moreover, no such AbA-resistance was found when the FBS1 sequence was changed to AATTA (mFBS1) within this construct. These data suggest that FLP can bind directly to the PIN3 promoter via FBS1.

We then tested whether this interaction also occurs in planta using ChIP-PCR analysis with anti-GR antibodies (Fig. 4d). FLP activity was controlled conditionally in the proFLP::FLP-GR/flp-1 $m y b 88$ line, and analysed for a differential enrichment at two different regions in the PIN3 promoter (amplicons PIN3_P1 and PIN3_P2) (Fig. 4a) as well as one in the unrelated ACTIN2 promoter. Compared to the mock treatment, a 6-h DEX treatment strongly enriched the amplicon closest to FBS1 (PIN3_P1), whereas DEX had no impact on the enrichment of PIN3_P2 and ACTIN2 (Fig. 4d). These data further confirm that FLP is a direct regulator of PIN3 expression, a finding that was corroborated by Wang et al. ${ }^{34}$.

However, it has been reported that auxin-inducible PIN3 expression does not require de novo protein synthesis ${ }^{6,17}$,

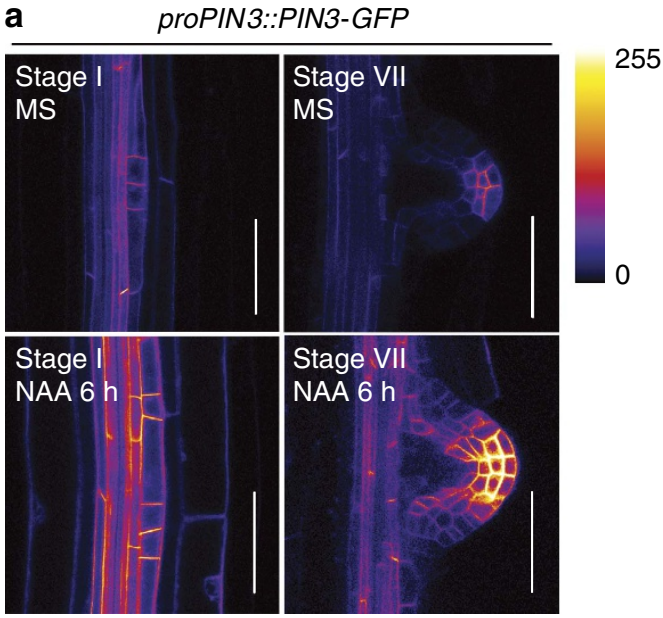

b proPIN3::PIN3-GFP/ flp-1 myb88
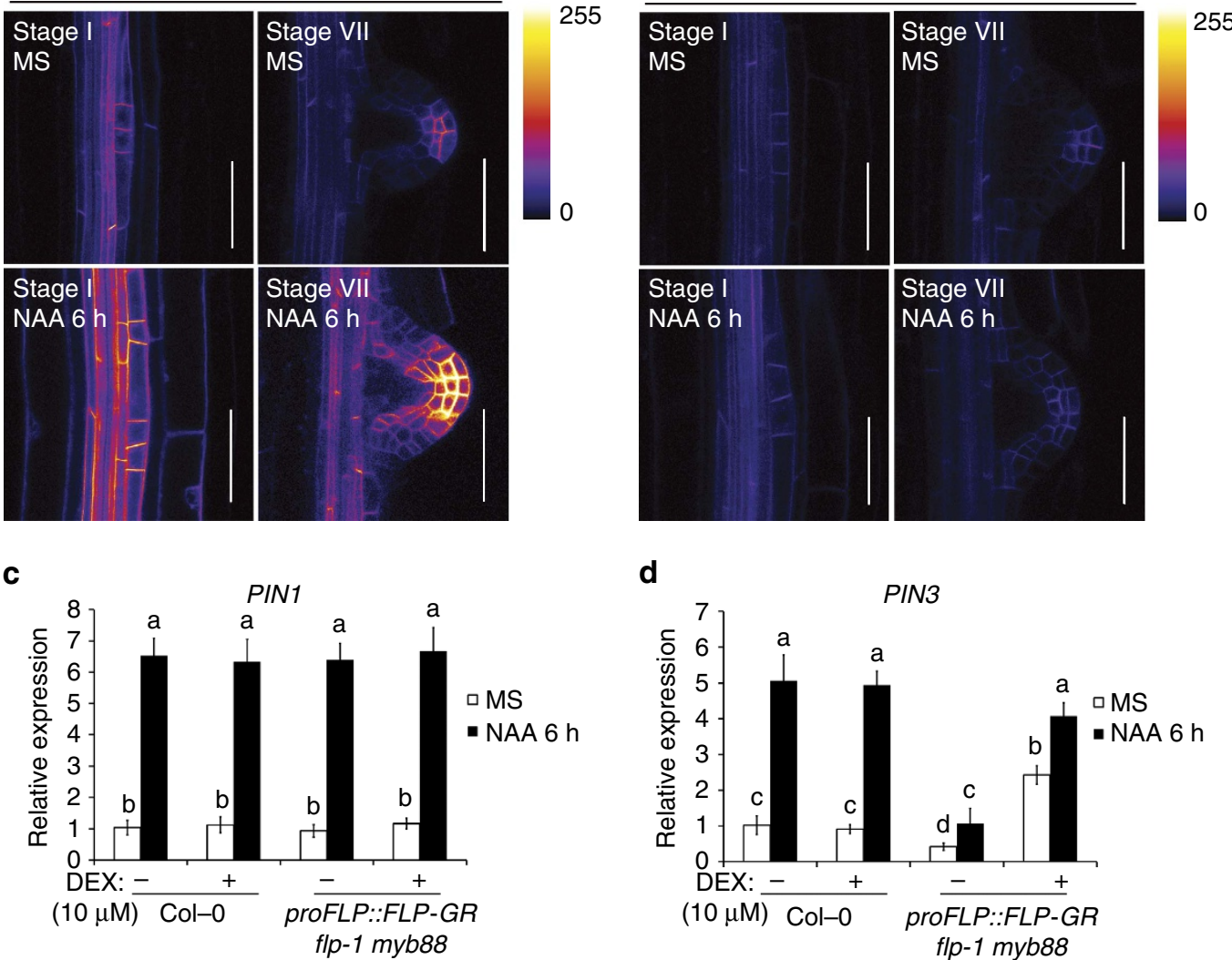

Figure 3 | FLP controls PIN3 expression. (a,b) Expression pattern of proPIN3::PIN3-GFP in (a) WT and (b) flp-1 myb88 in early stage (LR initiation, left panel) and later stage (stage VII, right panel) of LR with or without $6 \mathrm{~h}$ auxin treatments (10 $\mu \mathrm{M}$ NAA). Images were taken using identical confocal settings for all samples. Scale bar, $50 \mu \mathrm{m}$. Relative intensities are colour coded. (c,d) Relative auxin-inducible expression (6 h of $10 \mu \mathrm{M}$ NAA) of (c) PIN1 and (d) PIN3 determined by qRT-PCR from roots of WT and proFLP::FLP-GR/flp-1 myb88 seedlings ( $\pm 10 \mu M$ DEX for $6 \mathrm{~h}$ ). Data shown are means \pm s.d. and are representative of at least three independent experiments. Samples with different letters are significantly different: $P<0.05$ (Fisher's LSD mean separation test). 
a

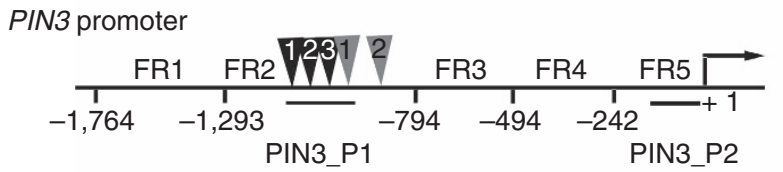

b

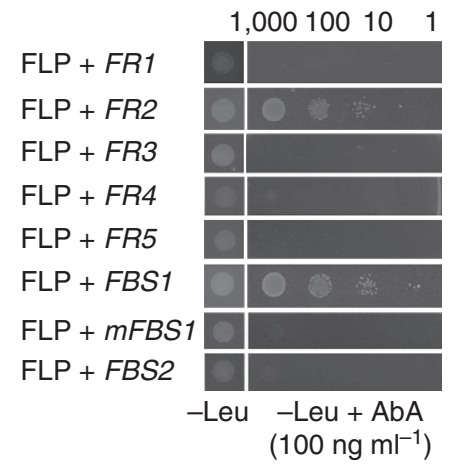

d

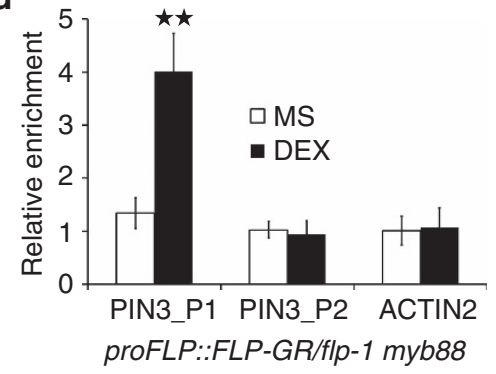

1 AuxRE1: GAGACA, $-1,128 /-1,123$
2 AuxRE2: TGTCTC, $-1,032 /-1,027$
3 AuxRE3: TGTCTC, $-1,016 /-1,011$
1 FBS1: AGCCG, -902 / -898
2 FBS2: TACCC, $-863 /-859$

C

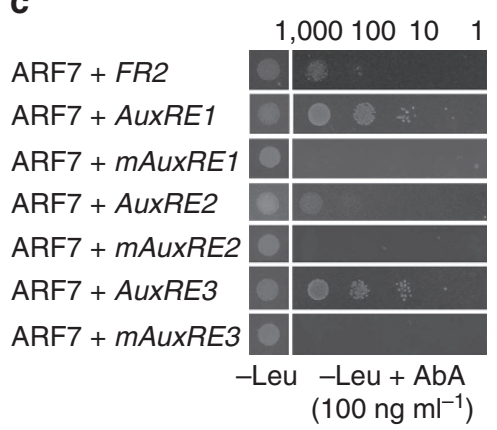

e

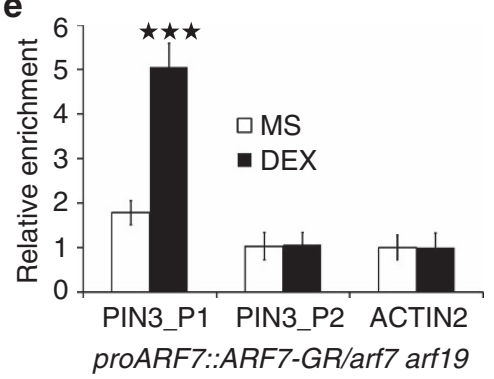

Figure 4 | FLP and ARF7 are direct regulators of PIN3 expression. (a) Schematic presentation of the PIN3 promoter (1.8 kb upstream of the translational start site at position ( + 1) with indication of the regions targeted for ChIP analysis (PIN3_P1 and PIN3_P2), the fragments used for yeast-one-hybrid (FR1-5) and the presence of auxin response elements (AuxREs, black triangles) and FLP binding sites (FBS, grey triangles) in fragment FR2. (b, c) Yeast-one-hybrid analysis of the interaction of (b) FLP with PIN3 promoter fragments (FR1-5), FBS1, a mutated FBS1 (mFBS1) and FBS2, and (c) of ARF7 with PIN3 promoter fragment FR2, its internal AuxREs (AuxRE1-3) and corresponding mutated versions (mAuxRE1-3). Left is yeast grown on SD-Leu medium in absence of Aureobasidin A ( $\mathrm{AbA}$ ). Right are the corresponding yeast strains in a dilution series grown on SD-Leu medium in the presence of $100 \mathrm{ng} \mathrm{ml}^{-1} \mathrm{AbA}$, a concentration where no autoactivation was detected for any of the strains. (d,e) Enrichment of the indicated DNA fragments (PIN3_P1, and PIN3_P2) following ChIP using anti-GR antibodies with (d) proFLP::FLP-GR/fIp-1 myb88 or (e) proARF7::ARF7-GR/arf7 arf19 treated for $6 \mathrm{~h}$ with or without DEX. A fragment from the ACTIN2 promoter was tested as a negative control. Data are presented as means \pm s.d. ${ }^{\star \star} P<0.01,{ }^{\star \star \star} P<0.001$ (Student's $t$-test). The experiment was repeated three times with similar results.

suggesting that PIN3 is a primary auxin-responsive gene, thus not requiring FLP as an intermediate regulator. Interestingly, the PIN3 promoter contains three 'canonical' auxin responsive elements (AuxREs) ${ }^{35,36}$, all of which are located within FR2, in close proximity to FBS1 (Fig. 4a). Using yeast-one-hybrid assays we found that ARF7 can interact with FR2 (Fig. 4c). In addition, short fragments spanning the individual AuxREs, also interacted with ARF7, and these interactions were lost when the AuxRE sequences were mutated (mAuxRE). We then tested whether ARF7 interacts with the PIN3 promoter in planta via ChIP-PCR in proARF7::ARF7-GR/arf7 arf19. In the absence of DEX, PIN3_P1 was only mildly enriched compared with PIN3_P2 and ACTIN2 (Fig. 4e). By contrast, the PIN3_P1 amplicon became strongly enriched when plants were treated with DEX, but DEX treatment did not stimulate the enrichment of PIN3_P2 and ACTIN2, confirming that PIN3 is indeed a primary auxin responsive gene. Thus our data demonstrate that PIN3 is a direct target of both ARF7 and FLP, two transcription factors that are required for auxin-responsive PIN3 transcription.

PIN3-driven LR development requires ARF7 and FLP binding. Next, we evaluated the role of FLP- and/or ARF7-mediated regulation of PIN3 on LR development. For this purpose, we designed proPIN3 variants of $1.8 \mathrm{~kb}$, containing specific mutations predicted to abrogate activation by FLP $(\mathrm{mF})$ or by ARF7 (mA), then fused them to PIN3-YFP and transformed them into pin3-4 (Fig. 5). Five independent lines were selected per construct, to minimize user-biased pre-selection for transgenic lines, and YFP positive individuals were analysed for auxin-responsive PIN3-YFP amplitude and LR density. In lines expressing PIN3-YFP from the reference PIN3 promoter (WT) a 5-8-fold higher PIN3-YFP expression was measured after auxin treatment, an amplitude within the order of magnitude of what is typically observed for endogenous PIN3 (Fig. 5a). Although strongly reduced, the proPIN3::PIN3-YFP variants impaired in either FLP or ARF7 recruitment $(\mathrm{mF}$ or $\mathrm{mA})$ still showed significant auxin responsive activity. When recruitment of both FLP and ARF7 was impaired $(\mathrm{mF}+\mathrm{mA})$, auxin could no longer up-regulate PIN3YFP expression significantly. These results are consistent with a model where FLP and ARF7 constitute the core mechanism by which auxin activates PIN3 expression.

These lines thus allowed us to probe the functional importance of FLP- and/or ARF7- regulated PIN3 expression for LR development. Therefore, we analysed the ability of these 


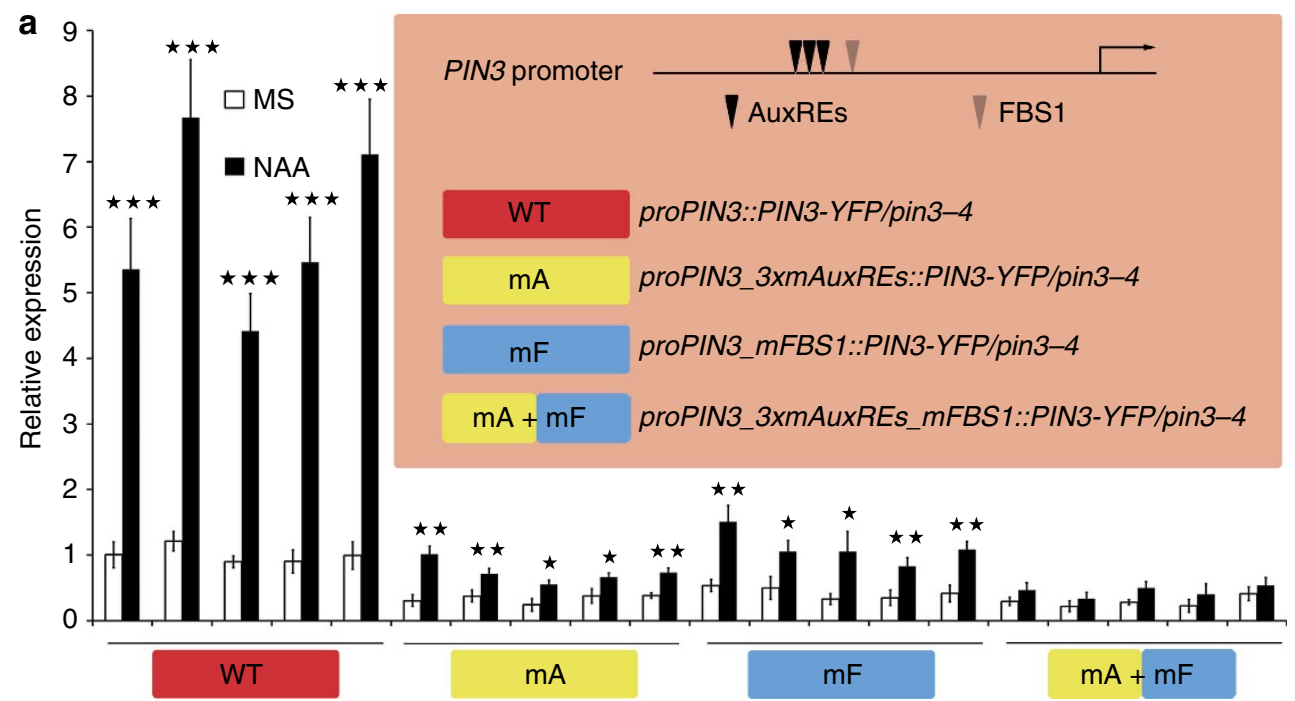

b
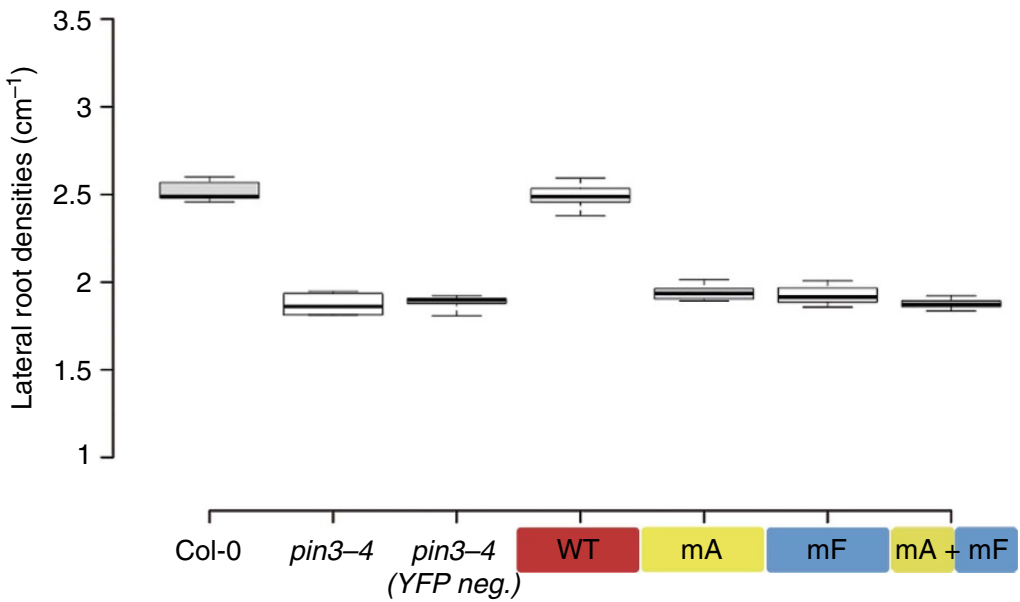

Figure 5 | In planta assessment of the relevance of ARF7 and FLP recruitment on auxin-inducibility of PIN3 and PIN3-dependent LR development. (a) Relative auxin-inducibility $(10 \mu \mathrm{M}$ NAA for $6 \mathrm{~h})$ of PIN3-YFP driven by the indicated proPIN3 variants. Roots of YFP positive plants were used for qRT-PCR assay. Scheme of PIN3 promoter illustrating by colour-coding the variants of the PIN3 constructs used to complement the pin3-4 mutant. Data are means \pm s.d. of at least three independent experiments. Asterisks denote Student's $t$-test significance: ${ }^{\star} P<0.05$, ${ }^{\star \star} P<0.01$ and ${ }^{\star \star \star} P<0.001$. (b) Quantification of the LR densities in 8-day-old seedlings of Col-0, pin3-4, YFP-negatives segregating among transgenic plants (proPIN3_WT), proPIN3_WT, proPIN3_mA, proPIN3_mF and proPIN3_mA + mF. Box plot description: centre lines show the medians of the averages of five independent transgenic lines, calculated for at least 20 individual plants per line; box limits indicate the 25th and 75th percentiles as determined by $\mathrm{R}$ software; whiskers extend to minimum and maximum values. $n=5$.

constructs to complement the LR defect in pin3-4. When PIN3-YFP was expressed under the control of the reference PIN3 promoter (WT), the LR density was restored to WT (Col-0) levels, whereas segregating YFP negative plants still showed the pin3-4 LR defect (Fig. 5b). In contrast, none of the mutant PIN3 promoter constructs were able to rescue the pin3-4 LR defect. Importantly, while mutations in either FLP or ARF7 binding sites still showed some PIN3-YFP auxin-mediated upregulation, this auxin-responsive PIN3-YFP expression was not sufficient to complement the LR defect in pin3-4. These results demonstrate that the regulation of auxin-sensitive PIN3 expression as defined by FLP as well as ARF7 is a crucial parameter for LR development.

Model of ARF7- and FLP-dependent PIN3 transcription. To probe how the joint activity of ARF7 and FLP impacts on auxin-regulated PIN3 transcription, we developed a mathematical model that integrates ARF7 and FLP into a coherent feed-forward motif (FFM) regulating PIN3 transcription and PIN3-mediated auxin transport (Fig. 6a). In parallel, we tested a model that lacks FLP function (mimicking a flp mutation) (Fig. 6b). A full description of the model and the parameter settings used can be found in the Methods section. Our simulation results indicate that the FFM may function to prolong and amplify PIN3 expression in response to auxin stimuli (Fig. $6 c-h$ ). In contrast to the system lacking the FFM, PIN3 in the FFM model continues to be actively transcribed for several hours upon removal of the auxin stimulus. Such a delay in response shutdown after stimulus removal is thought to be a key feature of coherent OR-type feedforward circuits ${ }^{37}$. Simulations with other parameter settings gave qualitatively similar results (see Supplementary Figs 6-9). To test the sensitivity of the observed PIN3 response delay to variations in the model parameters, we screened the ratio of the total PIN3 protein concentration response time upon auxin stimulus removal for model circuits with and without FFM as a function of single parameter changes (see Methods and Supplementary Fig. 10). For none of the parameters screened, the FFM/no FFM response time ratio drops below 1 in the parameter range 
a

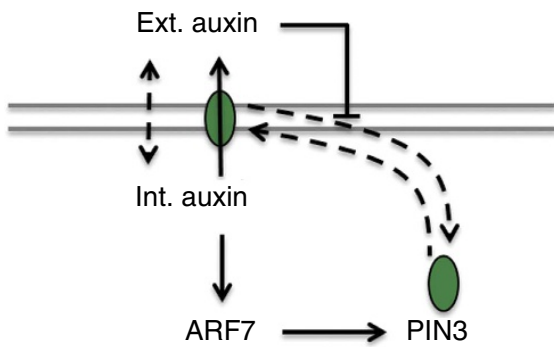

$\longleftrightarrow$ FLP

c

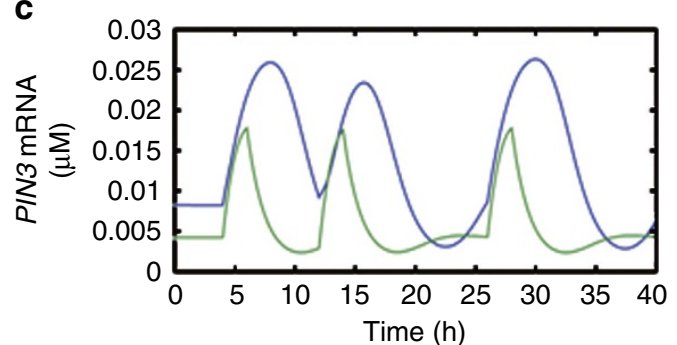

e

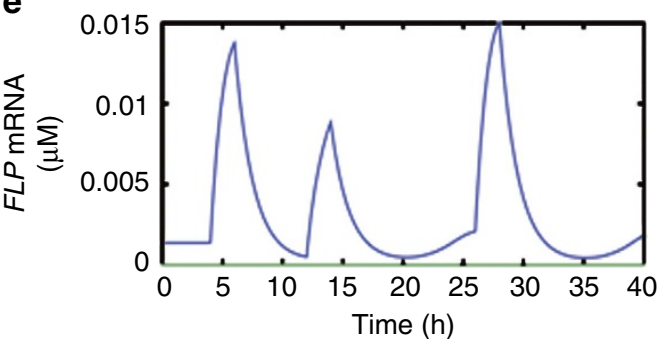

g

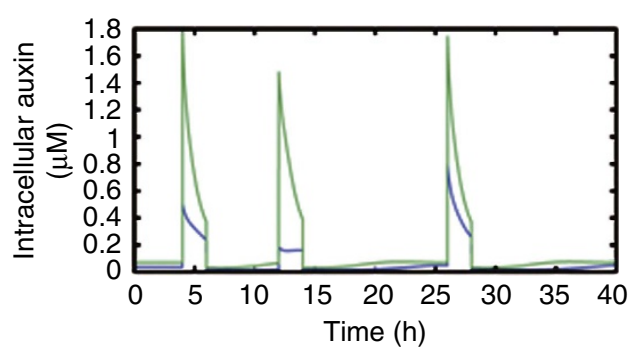

b

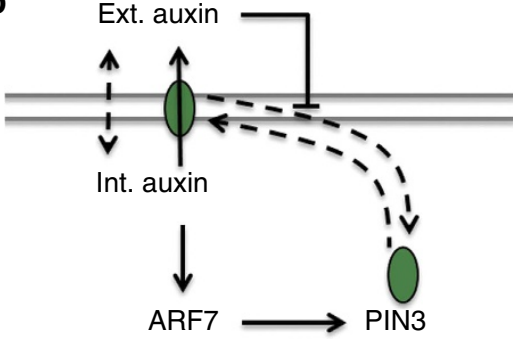

d

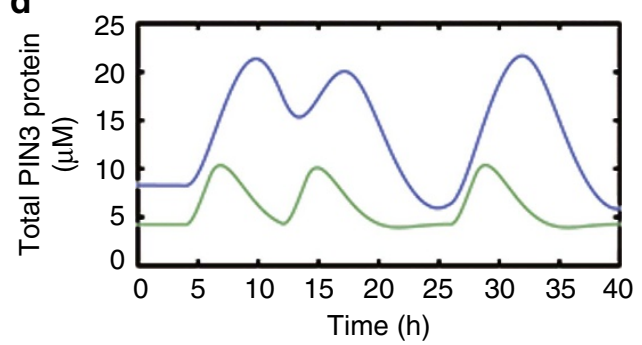

f

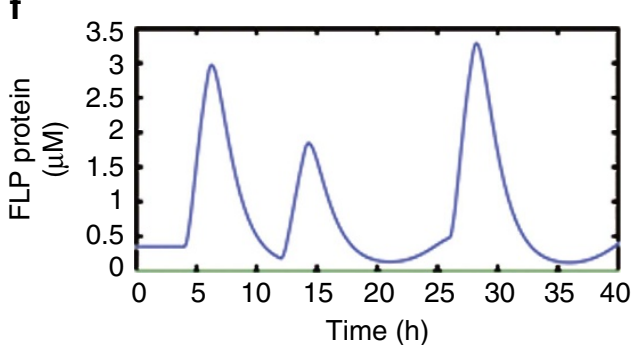

h

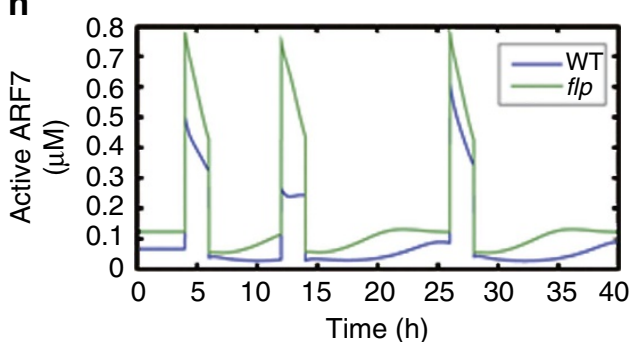

Figure 6 | Simulation of ARF7 and FLP-regulated PIN3 dynamics. (a) The coherent feed-forward motif (FFM) scheme and (b) the no-FFM scheme used to model auxin-induced PIN3 transcription. (c-h) Simulations of a PIN3-dependent auxin transport model subject to extracellular auxin pulses. Simulations were performed on model systems with (blue) and without (green) a transcriptional FFM involving FLP (see Methods), using the parameter settings given in Supplementary Table 1. Two-hour long $1 \mu \mathrm{M}$ auxin pulses were given to the system at $t=4 \mathrm{~h}, t=12 \mathrm{~h}$ and $t=26 \mathrm{~h}$. The subplots depict the resulting dynamics of PIN3 mRNA (c), total PIN3 protein, that is, the sum of intracellular and membrane-bound PIN3 (d), FLP mRNA (e), FLP protein (f), intracellular auxin (g) and active ARF7 (h) concentrations over time. The model simulations indicate that the FLP feed-forward circuit causes prolonged transcriptional activation of PIN3, resulting in an enhanced memory effect by which auxin stimulation reduces the impact of subsequent extracellular auxin stimuli on ARF7 activity and downstream signalling for a prolonged period of time. The system dynamics are qualitatively independent of the exact parameter settings used (see Supplementary Figs 6-10).

profiled, indicating that PIN3 response delay upon auxin stimulus removal is a robust feature of the circuit incorporating the FLP FFM. As expected, the response time ratio approximates 1 when FFM parameters approach values that make the FFM non-functional, for example, very-low FLP mRNA or protein synthesis rates $\left(\alpha_{\mathrm{FLP}}\right.$ or $\left.\beta_{\mathrm{FLP}}\right)$, very-high FLP mRNA or protein decay rates $\left(\gamma_{\mathrm{FLP}}\right.$ or $\left.\delta_{\mathrm{FLP}}\right)$, or very-high half-max constants for FLP-dependent activation of PIN3 transcription or ARF7-dependent activation of FLP transcription $\left(K_{\mathrm{FP}}\right.$ or $\left.K_{\mathrm{AF}}\right)$. Long PIN3 mRNA or protein half-lives (small $\gamma_{\mathrm{PIN} 3}$ or $\delta_{\mathrm{PIN} 3}$ ) also appear to offset the effect of the FFM (see Supplementary Fig. 10).
In the present context, we speculate that the FFM regulation of auxin signalling provides a temporary memory of cellular auxin levels to specific specialized cells, potentially enhancing the robustness of auxin flux patterns and focussing auxin maxima during post-embryonic patterning processes, such as LR initiation and development. In addition, our simulations indicate that delayed PIN3 down-regulation might temporarily capacitate the system for mitigating the effects of subsequent auxin stimuli on ARF7 activity and downstream auxin signalling (Fig. 6h). Together our data represent a first example of a mechanism by which plant endogenous cues such as auxin can become 
'memorized' to temporarily sustain transcriptional auxin signalling in selected cells for specific transcripts.

\section{Discussion}

Local auxin accumulation serves an essential role in triggering transcriptional changes associated with developmental transitions during plant organogenesis, as is reflected during LR development. A remarkable feature of auxin transport orchestrating organogenesis and regeneration, is the multi-level feed-back regulation of PIN-mediated auxin transport flows that are reinforced by the hormone itself ${ }^{8}$. Prominently among them is the auxin-induced transcription of $P I N s^{17}$, stimulating the cellular auxin transport capacity. Although not studied in detail, it has been generally accepted that the auxin-sensitive expression of several PINs, including PIN3, is directly activated by the canonical auxin signalling ${ }^{6,17}$. Our data further elaborate this notion by demonstrating that direct binding of ARF7 to the PIN3 promoter is critical for auxin-sensitive transcription of PIN3.

Such auxin-dependent regulation might mainly act to amplify the complex, developmentally wired cis-regulatory architectures that underlie the tissue-specific expression patterns of the different $P_{I N s^{38}}$. Here, we propose that the auxin-sensitive expression of PIN3 depends on binding of both ARF7 and FOUR LIPS, a MYB transcription factor previously characterized for its role in guard cell development. In addition, we found that ARF7 also controls auxin-sensitive FLP expression, thus defining a coherent FFM for auxin-induced PIN3 expression. Mathematical simulations revealed that this configuration could amplify the auxin-response output of PIN3 transcription and also temporarily sustain elevated PIN3 transcription after the auxin-stimulus is removed. This could allow for depletion of cellular auxin below the levels that were initially needed to activate PIN3 transcription, and for temporal buffering of random auxin fluctuations. This enhanced auxin transport capacity seems to stimulate FCs to proceed into LR initiation. Such transcriptional control mechanisms could also be relevant for developmental processes that are associated with auxin depletion, such as fruit valve specification $^{21,39}$ and stomatal development and patterning ${ }^{24}$. Interestingly, coherent FFMs of the type described here are abundantly found in transcriptional networks in yeast, animals and plants ${ }^{40-43}$, suggesting that this motif might represent an evolutionarily favoured solution to obtain a response delay upon stimulus shutdown.

Our data suggest that this auxin-sensing circuitry of PIN3 expression is involved LR initiation. In this developmental process, the high auxin concentrations that promote the progression from FC to LRI depends in part on PIN3-mediated auxin $\operatorname{transport}^{12}$, as illustrated by the diminished DR5 expression in flp myb88 LR primordia. Similar transcriptional architectures could underlie the auxin-sensitive expression of other PINs, including PIN1 and PIN4 that were found to be directly controlled by the auxin-inducible MADS-box XAL2/AGL14 (ref. 22), and PIN7 that was also found to be directly regulated by FLP binding to its promoter ${ }^{34}$.

\footnotetext{
Methods

Plant materials and growth conditions. Arabidopsis seeds were surface-sterilized for $15 \mathrm{~min}$ in $10 \%$ bleach, washed four times with sterile water, and plated on half-strength Murashige and Skoog medium ( $0.8 \%$ agar). Plants were stratified at $4{ }^{\circ} \mathrm{C}$ for 2 days in darkness and then transferred to a growth chamber at $22^{\circ} \mathrm{C}$ under continuous illumination (light intensity $120 \mu \mathrm{mol} \mathrm{m}^{-2} \mathrm{~s}^{-1}$ ).

Arabidopsis thaliana ecotypes Columbia (Col-0), and Ler were used as controls. The following lines/seeds/constructs were used in this study: $f l p-1$ (ref. 44 ), $f l p-7$ (ref. 25), myb88 (SALK_068691) ${ }^{25}, f l p-1$ myb88 (ref. 25), flp-7 myb88 (ref. 25), proFLP::GUS-GFP ${ }^{25}$, cyca2;234 (ref. 26), flp-7 myb88 cyca2;234 (ref. 26), slr-1 (ref. 32), proARF7::ARF7-GR/nph4-1(arf7)arf19-1 (ref. 33), pin3-4 (SALK_038609) ${ }^{45}$, proDR5::GUS ${ }^{46}$, proDR5rev::GFP ${ }^{47}$ proPIN3::PIN3-GFP ${ }^{45}$. Published mutants and
}

reporter lines were crossed into $f l p-1$ myb88 and $f l p-7$ myb88. proFLP::FLP-GR/flp-1 myb88, proFLP::NLS-GFP, proPIN3::PIN3-YPF/pin3-4 lines were generated through Agrobacterium-mediated floral dip transformation ${ }^{48}$

GUS staining and microscopy. The $\beta$-glucuronidase (GUS) assays were performed as follows: seedlings were incubated in GUS staining buffer $(0.1 \mathrm{M}$ Tris pH7.5 containing $\left.2.9 \mathrm{mg} \mathrm{ml}^{-1} \mathrm{NaCl}, 6.6 \mathrm{mg} \mathrm{ml}^{-1} \mathrm{~K}_{3} \mathrm{Fe}(\mathrm{CN})_{6}\right)$ at $37^{\circ} \mathrm{C}$ overnight $^{49}$. For microscopic analysis, samples were cleared by mounting in lactic acid (Acros Organics, Geel, Belgium) or were transferred to $0.24 \mathrm{~N} \mathrm{HCl}$ in $20 \%$ at $57^{\circ} \mathrm{C}$ for $15 \mathrm{~min}$. This solution was replaced with $7 \% \mathrm{NaOH}, 7 \%$ hydroxylamine- $\mathrm{HCl}$ in $60 \%$ ethanol for $15 \mathrm{~min}$ at room temperature. Roots were then rehydrated for $5 \mathrm{~min}$ each in 40, 20 and 10\% ethanol, and infiltrated for $15 \mathrm{~min}$ in 5\% ethanol, 25\% glycerol. Roots were mounted in $50 \%$ glycerol on glass microscope slides ${ }^{50}$. All samples were analysed using a BX53 Olympus microscope. Fluorescence images were acquired by a Zeiss 710 confocal laser scanning microscope equipped with a C-Apochromat $\times 40$ water immersion objective. If needed for visualization purposes, further processing was done using Image J software by uniformly changing brightness and contrast to the entire image per channel. For the images in Fig. 1a, the smoothing function was applied to both channels.

Phenotyping and statistics. To analyse stages of LR development, about 20 roots from 6-day-old seedlings were processed per data point and genotype. Plant material was cleared following the more elaborate protocol as described above ${ }^{50}$. Root lengths were measured with the Image J software (NIH; http:// rsb.info.nih.gov/ij). LRPs were counted with a differential interference contrast microscope (BX53; Olympus). LRP density was calculated as the ratio of the total number of LRP over the sum of root length. Phenotypic analysis of FC and LRP was done as described ${ }^{12}$. In brief, 10 roots of 5-day-old seedling (grown at $21^{\circ} \mathrm{C}$ $16 \mathrm{~h}$ light $8 \mathrm{~h}$ dark) were analysed per experiment. The counting of FCs and LRP was performed in the direction from root tip towards the root base. Zeiss LSM 710 confocal microscope with a $\times 40$ (water immersion) objective with GFP settings (excitation $488 \mathrm{~nm}$, emission $507 \mathrm{~nm}$ ) were used to identify and count DR5rev::GFP-positive signals in the pericycle. GFP signals accompanied with nuclear divisions were scored as LRP, whereas FCs were counted when no cell divisions were observed. The data are represented as averages of three independent experiments, unless indicated differently. The statistical significance was evaluated by Student's $t$-test analysis. For multiple comparisons, an analysis of variance followed by Fisher's Least Significant Difference (LSD) mean separation test (SPSS) was performed on the data. Samples with different letters are significantly different at $P<0.01$ or $P<0.05$. Boxplots were generated using BoxPlotR ${ }^{51}$.

Cloning. To construct proFLP::FLP-GR, a FLP promoter region $( \pm 3 \mathrm{~kb}$ upstream of translational start) was amplified with primers proFLP-F and proFLP-R and cloned into pDONR ${ }^{\mathrm{TM}} \mathrm{P} 4-\mathrm{P} 1 \mathrm{R}$. The FLP cDNA was amplified with primers FLP-F and FLP-R and cloned into $\mathrm{pDONR}^{\mathrm{TM}} 221$; The above-mentioned pDONR vectors were subcloned together with a Glucocorticoid ORF containing (pDONR ${ }^{\mathrm{TM}} \mathrm{P} 2 \mathrm{R}$ P3) into pB7m34GW 52,53 with MultiSite Gateway Three-Fragment Vector Construction Kit (Invitrogen). cDNA of ARF7 was PCR amplified with primers ARF7-F and ARF7-R, and cloned into pDONR ${ }^{\mathrm{TM}}$ 221. proFLP in $\mathrm{pDONR}^{\mathrm{TM}} \mathrm{P} 4-$ P1R was subcloned into pEX-K7SNFm14GW to construct proFLP::NLS-GFP.

A promoter $1.8 \mathrm{~kb}$ upstream of the PIN3 translational start was cloned into pDONR ${ }^{\mathrm{TM}} \mathrm{P} 4-\mathrm{P} 1 \mathrm{R}$ with primers proPIN3_FL_F and proPIN3_FL_R. Site-directed mutagenesis was performed using PCR to mutate AuxREs and FBS. The resulting promoters were cloned into pDONR ${ }^{\mathrm{TM}} \mathrm{P} 4-\mathrm{P} 1 \mathrm{R}$, and together with PIN3-YFP in

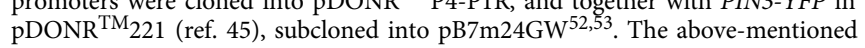
primers are listed in Supplementary Table 2.

Gene Expression Analysis by qRT-PCR. For qRT-PCR analysis, 6-day-old seedlings were treated with NAA $(10 \mu \mathrm{M})$ and/or DEX $(10 \mu \mathrm{M})$ at different time points and roots were harvested for RNA extraction. RNA was extracted with an RNeasy kit (QIAGEN). Poly(dT) cDNA was prepared from $1 \mu \mathrm{g}$ of total RNA with SuperScript III reverse transcriptase (Invitrogen) and analysed on a LightCycler 480 apparatus (Roche Diagnostics) with the SYBR Green I Master kit (Roche Diagnostics) according to the manufacturer's instructions. Primer pairs were designed with Beacon Designer 4.0 (Premier Biosoft International) (Supplementary Table 2). All individual reactions were performed as biological triplicates. Data was analysed with qBase ${ }^{54}$. Expression levels were normalized to those of ACTIN2. The statistical significance was evaluated by Student's $t$-test analysis for pair-wise comparisons and by Fisher's LSD means separation test (SPSS) for multiple comparisons.

ChIP-qPCR assay. Eight-day-old seedlings of proFLP::FLP-GR/flp-1 myb88 or proARF7::ARF7-GR/arf7 arf19 were treated with $10 \mu \mathrm{M}$ DEX for $6 \mathrm{~h}$. One gram root material per sample was used for ChIP experiments. Plant materials were cross-linked in $1 \%$ formaldehyde and their chromatin isolated. Anti-GR antibody ab3580 (abcam) (1:500) was used to immunoprecipitate the protein-DNA complex, and the precipitated DNA was purified using a PCR purification kit (Qiagen) for qRT-PCR analysis. The ChIP experiments were performed three times. Chromatin 
precipitated without antibody was used as negative control, while the isolated chromatin before precipitation was used as input control ${ }^{55,56}$. The enrichment of DNA fragments was determined by quantitative PCR using the following primer pairs: proACT2_F and proACT2_R; proFLP(P1)_F, proFLP(P1)_R, proFLP(P2)_F, proFLP(P2)_R, proPIN3(P1)_F and proPIN3(P1)_R; proPIN3(P2)_F and proPIN3(P2)_R (Supplementary Table 2).

Yeast-one hybrid. Yeast-one-hybrid assays were performed with the kit provided by Clontech (Matchmaker One-Hybrid Library Construction and Screening kit) using the Y1HGold yeast strain according to the manufacturer's instructions. This system detects binding events by resistance against the antibiotic Aureobasidin $\mathrm{A}(\mathrm{AbA})$.

The primers used for cloning the related cDNAs or promoter DNAs are listed

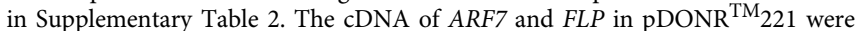
subcloned into pGADT7. The promoter fragments of PIN3 were cloned into the $\mathrm{SacI} / \mathrm{SalI}$ sites of pAbAi vector, and efficiently integrated into the genome of the Y1H Gold yeast strain by homologous recombination on SD-U medium. Matchmaker ${ }^{\mathrm{TM}}$ Insert Check PCR Mix was used to verify positive colonies. Autoactivation tests for the promoter were conducted in the range from 0 to $500 \mathrm{ng} \mathrm{ml}^{-1} \mathrm{AbA}$, and background activation was detected up to $50 \mathrm{ng} \mathrm{ml}^{-1}$. An AbA concentration of $100 \mathrm{ng} \mathrm{ml}^{-1}$ was used to screen positive colonies. Then pGADT7 prey vectors harboring ORF of $A R F 7$ or FLP were transformed into the above-mentioned yeast strain that have already integrated the promoter fragments of PIN3 or FLP. After having grown on SD-Leu for 2-3 days, the colonies able to grow on the selective medium were transferred to $100 \mu \mathrm{l}$ of sterile ultrapure water and spotted as $5 \mu \mathrm{l}$ droplets onto SD-Leu $+\mathrm{AbA}$ plates. Plates were incubated at $28^{\circ} \mathrm{C}$ for 3-4 days.

Modelling the effects of FLP on PIN3 and auxin dynamics. We mathematically modelled the impact of the FLP FFM on PIN3 expression dynamics starting from an earlier model by Jönsson et al. ${ }^{57}$. The revised model is defined by the following ordinary differential equations:

$$
\begin{gathered}
\frac{\mathrm{d} A_{\mathrm{int}}}{\mathrm{d} t}=\beta_{\mathrm{A}}-\delta_{\mathrm{A}} A_{\mathrm{int}} \\
+\frac{a}{V}\left[p_{\mathrm{AH}}\left(f_{\mathrm{AH}}^{\mathrm{wall}} A_{\mathrm{ext}}-f_{\mathrm{AH}}^{\mathrm{cell}} A_{\mathrm{int}}\right)\right. \\
\left.+p_{\mathrm{A}}-\frac{P I N 3_{\mathrm{mem}}}{R_{\mathrm{P}}}\left(f_{\mathrm{A}^{-}}^{\text {wall }} N_{\mathrm{influx}} \frac{K_{\mathrm{A}} A_{\mathrm{ext}}}{K_{\mathrm{A}}+A_{\mathrm{ext}}}-f_{\mathrm{A}^{-}}^{\text {cell }} N_{\mathrm{efflux}} \frac{K_{\mathrm{A}} A_{\mathrm{int}}}{K_{\mathrm{A}}+A_{\mathrm{int}}}\right)\right] \\
\frac{\mathrm{d} F L P_{\mathrm{mRNA}}}{\mathrm{d} t}=\alpha_{\mathrm{FLP}}\left(\frac{A R F 7_{\mathrm{act}}^{n_{2}}}{K_{\mathrm{AF}}^{n_{2}}+A R F 7_{\mathrm{act}}^{n_{2}}}\right)-\gamma_{\mathrm{FLP}} F L P_{\mathrm{mRNA}} \\
\left.\frac{\mathrm{d} F L P}{K_{\mathrm{ARF} 7}^{n_{1}}+A_{\mathrm{int}}^{n_{1}}}\right) \\
\frac{\mathrm{d} P I N 3_{\mathrm{mRNA}}}{\mathrm{d} t}=\beta_{\mathrm{FLP}} F L P_{\mathrm{mRNA}}-\delta_{\mathrm{FLP}} F L P \\
\frac{\mathrm{d} P I N 3}{\mathrm{~d} t}=\beta_{\mathrm{PIN} 3}\left(\frac{A R F 7_{\mathrm{act}}^{n_{3}} / K_{\mathrm{AP}}^{n_{3}}+F I N 3_{\mathrm{mRNA}}-\delta_{\mathrm{PIN} 3}^{n_{4}} / K_{\mathrm{FP}}^{n_{4}}}{1+A R F 7_{\mathrm{act}}^{n_{3}} / K_{\mathrm{AP}}^{n_{3}}+F L P^{n_{4}} / K_{\mathrm{FP}}^{n_{4}}}\right)-\gamma_{\mathrm{PIN} 3} P I N 3_{\mathrm{mRNA}} \\
k_{1} P I N 3+\frac{a}{V} k_{2} \frac{K^{n_{5}}}{K^{n_{5}}+A_{\mathrm{ext}}^{n_{5}} P I N 3_{\mathrm{mem}}}
\end{gathered}
$$

$$
\frac{\mathrm{d} P I N 3_{\mathrm{mem}}}{\mathrm{d} t}=\frac{V}{a} k_{1} P I N 3-k_{2} \frac{K^{n_{5}}}{K^{n_{5}}+A_{\mathrm{ext}}^{n_{5}}} P I N 3_{\mathrm{mem}}-\delta_{\mathrm{PIN} 3} P I N 3_{\mathrm{mem}}
$$

with $A_{\text {int }}$ and $A_{\text {ext }}$ the intra- and extracellular auxin concentrations, respectively, $A R F 7_{\text {act }}$ the concentration of active ARF7 protein, FLP $P_{\text {mRNA }}$ and FLP the concentrations of FLP mRNA and protein, PIN3 $3_{\mathrm{mRNA}}$ the concentration of PIN3 mRNA, and PIN3 and PIN $3_{\text {mem }}$ the concentrations of intracellular and membranelocalized PIN3 protein. $\alpha$ and $\beta$ parameters denote mRNA and protein (or auxin) production rates, respectively, and $\gamma$ and $\delta$ parameters denote the corresponding degradation rates. $K^{\prime}$ s indicate half-maximal activation constants, and $n^{\prime}$ s are activation curve Hill coefficients. Detailed parameter definitions and dimensions, as well as the default parameter values used, are presented in Supplementary Table 1 (refs 57-59).

As in Jönsson et al. ${ }^{57}$, the intracellular auxin concentration in our model is influenced by intracellular auxin production and degradation, and by passive and active auxin transport across the cell membrane. In contrast to Jönsson et al. ${ }^{57}$, the auxin dynamics in the cell wall are not modelled explicitly. Instead, the temporal profile of the extracellular auxin concentration $\left(A_{\text {ext }}\right)$ is predefined for each simulation, in terms of a constant background concentration and auxin pulses during specific epochs (see below). In equation (1), the saturability of $P I N 3_{\mathrm{mem}^{-}}$ mediated active auxin transport is modelled slightly differently from the original model ${ }^{57}$. In particular, $P I N 3_{\text {mem }} / R_{\mathrm{P}}$ (with $R_{\mathrm{P}}$ a reference $P I N 3_{\text {mem }}$ concentration) is used as a factor to modulate the membrane permeability $p_{\mathrm{A}}^{-}$, instead of limiting the maximum membrane PIN concentration to $1.0 \mu \mathrm{mol}$ per unit area as in ref. 57 .
In addition, the form of the auxin-dependent saturation characteristic was reverted to the form used earlier by Mitchison et al. ${ }^{58}$, which includes an additional $K_{\mathrm{A}}$ factor in the numerator of the Michaelis-Menten relation, to restore the unit balance. In equations (6) and (7), the formulation of auxin-dependent PIN3 membrane transport and PIN3 membrane localization has been changed to reflect the observation that auxin inhibits the internalization of membrane-bound PIN3 rather than stimulating PIN3 delivery to the plasma membrane $e^{60,61}$, and to better reflect the membrane concentration nature of $P I N 3_{\text {mem }}\left(\mu \mathrm{mol} \mathrm{dm}{ }^{-2}\right)$.

We complemented this auxin transport model with equations describing the dynamics of the transcriptional feed-forward circuit impacting PIN3 expression (Equations (2)-(6)). Assuming a constant ARF7 protein concentration $(A R F 7=1 \mu \mathrm{M})$, ARF7 activity kinetics are modelled as a combination of basal, auxin independent ARF7 activity $\left(\omega_{\mathrm{ARF}}\right)$ and auxin-dependent ARF7 activity (Equation (2)). Equation (3) describes how FLP mRNA levels are influenced by $A R F 7_{\text {act }}$-dependent transcription and mRNA degradation, while equation (4) describes FLP protein concentration changes as a function of protein production and degradation. Equation (5) incorporates the effects of active ARF7 and FLP on PIN3 transcription, as well as an mRNA degradation term. Equation (6) incorporates PIN3 protein production and degradation terms, in addition to the membrane transport-related terms discussed above. A protein degradation term was also added to equation (7), under the assumption that membrane-bound PIN3 is also earmarked for degradation.

Simulations were performed in MATLAB R2013b, using an implicit numerical solver with adaptable time step used for solving initial value problems for stiff ordinary differential equations (ode23s.m function). The MATLAB scripts are available from http://www.psb.ugent.be/esb/ESB/FLP_FFM_SI.html. The default simulations (Fig. 6c-h) were performed using the parameter settings described in Supplementary Table 1. mRNA and protein degradation rate constants were set to reflect typical mRNA and protein half-lives of $\sim 1 \mathrm{~h}$. In the default settings, FLP was set to have a shorter protein half-life $(30 \mathrm{~min})$ than PIN3 (2 h) and the half-max constants for FLP- and ARF7-dependent activation of PIN3 transcription $\left(K_{\mathrm{FP}}\right.$ and $K_{\mathrm{AP}}$ ) were set to 0.6 and $0.3 \mu \mathrm{M}$, respectively, in order to achieve a $\sim 50 \%$ drop in PIN3 expression level as observed in the flp mutant after $6 \mathrm{~h}$ (see Fig. $3 \mathrm{~d}$ ). Initial auxin, PIN3 and FLP levels were set to the steady-state levels obtained for a constant extracellular auxin concentration $\left(A_{\text {ext }}\right)$ of $0.1 \mu \mathrm{M}$. Simulations of the $f l p$ mutant were performed by setting $\alpha_{\mathrm{FLP}}, \beta_{\mathrm{FLP}}, \gamma_{\mathrm{FLP}}, \delta_{\mathrm{FLP}}$ and the initial FLP mRNA and protein concentrations to zero. In all simulations, a background extracellular auxin concentration of $0.1 \mu \mathrm{M}$ was assumed, and $2 \mathrm{~h}$-long $1 \mu \mathrm{M}$ extracellular auxin pulses were simulated at $t=4-6 \mathrm{~h}, t=12-14 \mathrm{~h}$ and $t=26-28 \mathrm{~h}$.

PIN3 response delay upon stimulus removal to parameters. Although the parameters listed in Supplementary Table 1 are within biologically reasonable ranges, the resulting model cannot be taken to be quantitatively accurate, as this would require quantitative measurements of all model parameters and absolute molecular concentration ranges in vivo. To test the robustness of our modellingbased conclusions, we performed a series of simulations screening the effects of parameter changes on the qualitative dynamics of the FFM versus no-FFM circuits. In each simulation, one parameter was changed from its default value listed in Supplementary Table 1, while the other parameters were kept at their default values. Hill coefficients $n_{1}, n_{2}, n_{3}, n_{4}, n_{5}$ were screened in the range $[1,3], \omega_{\text {ARF7 }}$ was screened in the range $[0,1]$ and the other parameters were screened in a range encompassing one order of magnitude above and below the default values listed in Supplementary Table 1 (with the exception of $\beta_{\mathrm{A}}$, the midrange value of which (on log scale) was set to $5 \times 10^{-6} \mathrm{~s}^{-1}$ instead of $0 \mathrm{~s}^{-1}$ for screening purposes). The resulting alternatively parameterized circuits were simulated with and without FFM under a $1 \mu \mathrm{M}$ extracellular auxin stimulus until steady-state was reached, after which the auxin stimulus was shut down and the PIN3 protein response time was measured, defined as the time it takes the system to bridge half of the PIN3 protein concentration difference between the auxin-stimulated and unstimulated steady states. FFM/no FFM response time ratios $>1$ indicate that the circuit with FFM exhibits a delayed PIN3 protein response upon auxin stimulus shutdown.

\section{References}

1. De Smet, I. et al. Auxin-dependent regulation of lateral root positioning in the basal meristem of Arabidopsis. Development 134, 681-690 (2007).

2. Moreno-Risueno, M. A. et al. Oscillating gene expression determines competence for periodic Arabidopsis root branching. Science 329, 1306-1311 (2010).

3. Van Norman, J. M., Xuan, W., Beeckman, T. \& Benfey, P. N. To branch or not to branch: the role of pre-patterning in lateral root formation. Development 140, 4301-4310 (2013).

4. Vermeer, J. E. et al. A spatial accommodation by neighboring cells is required for organ initiation in Arabidopsis. Science 343, 178-183 (2014).

5. Lucas, M. et al. Lateral root morphogenesis is dependent on the mechanical properties of the overlaying tissues. Proc. Natl Acad. Sci. USA 110, 5229-5234 (2013).

6. Péret, B. et al. Sequential induction of auxin efflux and influx carriers regulates lateral root emergence. Mol. Syst. Biol. 9, 699 (2013).

7. Kumpf, R. P. et al. Floral organ abscission peptide IDA and its HAE/HSL2 receptors control cell separation during lateral root emergence. Proc. Natl Acad. Sci. USA 110, 5235-5240 (2013). 
8. Vanneste, S. \& Friml, J. Auxin: a trigger for change in plant development. Cell 136, 1005-1016 (2009).

9. Lavenus, J. et al. Lateral root development in Arabidopsis: fifty shades of auxin. Trends Plant Sci. 18, 450-458 (2013).

10. Xuan, W. et al. Root cap-derived auxin pre-patterns the longitudinal axis of the Arabidopsis root. Curr. Biol. 25, 1381-1388 (2015).

11. Dubrovsky, J. G. et al. Auxin acts as a local morphogenetic trigger to specify lateral root founder cells. Proc. Natl Acad. Sci. USA 105, 8790-8794 (2008).

12. Marhavý, P. et al. Auxin reflux between the endodermis and pericycle promotes lateral root initiation. EMBO J. 32, 149-158 (2013).

13. Benková, E. et al. Local, efflux-dependent auxin gradients as a common module for plant organ formation. Cell 115, 591-602 (2003).

14. Swarup, K. et al. The auxin influx carrier LAX3 promotes lateral root emergence. Nat. Cell Biol. 10, 946-954 (2008).

15. Péret, B. et al. Auxin regulates aquaporin function to facilitate lateral root emergence. Nat. Cell Biol. 14, 991-998 (2012).

16. Petrášek, J. et al. PIN proteins perform a rate-limiting function in cellular auxin efflux. Science 312, 914-918 (2006).

17. Vieten, A. et al. Functional redundancy of PIN proteins is accompanied by auxin-dependent cross-regulation of PIN expression. Development 132 4521-4531 (2005)

18. Vernoux, T. et al. The auxin signalling network translates dynamic input into robust patterning at the shoot apex. Mol. Syst. Biol. 7, 508 (2011).

19. Han, M. et al. Structural basis for the auxin-induced transcriptional regulation by Aux/IAA17. Proc. Natl Acad. Sci. USA 111, 18613-18618 (2014)

20. Pierre-Jerome, E., Jang, S. S., Havens, K. A., Nemhauser, J. L. \& Klavins, E. Recapitulation of the forward nuclear auxin response pathway in yeast. Proc. Natl Acad. Sci. USA 111, 9407-9412 (2014).

21. Lavenus, J. et al. Inference of the Arabidopsis lateral root gene regulatory network suggests a bifurcation mechanism that defines primordia flanking and central zones. Plant Cell 27, 1368-1388 (2015).

22. Garay-Arroyo, A. et al. The MADS transcription factor XAL2/AGL14 modulates auxin transport during Arabidopsis root development by regulating PIN expression. EMBO J. 32, 2884-2895 (2013).

23. Cui, D. et al. The Arabidopsis IDD14, IDD15, and IDD16 cooperatively regulate lateral organ morphogenesis and gravitropism by promoting auxin biosynthesis and transport. PLoS Genet. 9, e1003759 (2013).

24. Le, J. et al. Auxin transport and activity regulate stomatal patterning and development. Nat. Commun. 5, 3090 (2014).

25. Lai, L. B. et al. The Arabidopsis R2R3 MYB proteins FOUR LIPS and MYB88 restrict divisions late in the stomatal cell lineage. Plant Cell 17, 2754-2767 (2005).

26. Vanneste, S. et al. Developmental regulation of CYCA2s contributes to tissue-specific proliferation in Arabidopsis. EMBO J. 30, 3430-3441 (2011).

27. Xie, Z. et al. Regulation of cell proliferation in the stomatal lineage by the Arabidopsis MYB FOUR LIPS via direct targeting of core cell cycle genes. Plant Cell 22, 2306-2321 (2010).

28. Yang, K. et al. Requirement for A-type cyclin-dependent kinase and cyclins for the terminal division in the stomatal lineage of Arabidopsis. J. Exp. Bot. 65, 2449-2461 (2014).

29. De Rybel, B. et al. A novel Aux/IAA28 signaling cascade activates GATA23dependent specification of lateral root founder cell identity. Curr. Biol. 20, 1697-1706 (2010).

30. De Smet, I. et al. Receptor-like kinase ACR4 restricts formative cell divisions in the Arabidopsis root. Science 322, 594-597 (2008).

31. Fukaki, H., Nakao, Y., Okushima, Y., Theologis, A. \& Tasaka, M. Tissue-specific expression of stabilized SOLITARY-ROOT/IAA14 alters lateral root development in Arabidopsis. Plant J. 44, 382-395 (2005).

32. Fukaki, H., Tameda, S., Masuda, H. \& Tasaka, M. Lateral root formation is blocked by a gain-of-function mutation in the SOLITARY-ROOT/IAA14 gene of Arabidopsis. Plant J. 29, 153-168 (2002).

33. Okushima, Y., Fukaki, H., Onoda, M., Theologis, A. \& Tasaka, M. ARF7 and ARF19 regulate lateral root formation via direct activation of LBD/ASL genes in Arabidopsis. Plant Cell 19, 118-130 (2007).

34. Wang, H. et al.Transcriptional regulation of PINs by FOUR LIPS and MYB88 during Arabidopsis root gravitropism. Nat. Commun. 6, 8822 (2015).

35. Ulmasov, T., Liu, Z. B., Hagen, G. \& Guilfoyle, T. J. Composite structure of auxin response elements. Plant Cell 7, 1611-1623 (1995).

36. Boer, D. R. et al. Structural basis for DNA binding specificity by the auxin-dependent ARF transcription factors. Cell 156, 577-589 (2014).

37. Mangan, S. \& Alon, U. Structure and function of the feed-forward loop network motif. Proc. Natl Acad. Sci. USA 100, 11980-11985 (2003).

38. Tanaka, H., Dhonukshe, P., Brewer, P. B. \& Friml, J. Spatiotemporal asymmetric auxin distribution: a means to coordinate plant development. Cell. Mol. Life Sci. 63, 2738-2754 (2006).

39. Sorefan, K. et al. A regulated auxin minimum is required for seed dispersal in Arabidopsis. Nature 459, 583-586 (2009).

40. Lee, T. I. et al. Transcriptional regulatory networks in Saccharomyces cerevisiae. Science 298, 799-804 (2002)
41. Alon, U. Network motifs: theory and experimental approaches. Nat. Rev. Genet 8, 450-461 (2007).

42. Le, D. H. \& Kwon, Y. K. A coherent feedforward loop design principle to sustain robustness of biological networks. Bioinformatics 29, 630-637 (2013).

43. Carrera, J., Rodrigo, G., Jaramillo, A. \& Elena, S. F. Reverse-engineering the Arabidopsis thaliana transcriptional network under changing environmental conditions. Genome Biol. 10, R96 (2009).

44. Yang, M. \& Sack, F. D. The too many mouths and four lips mutations affect stomatal production in Arabidopsis. Plant Cell 7, 2227-2239 (1995).

45. Zádníková, P. et al. Role of PIN-mediated auxin efflux in apical hook development of Arabidopsis thaliana. Development 137, 607-617 (2010).

46. Ulmasov, T., Murfett, J., Hagen, G. \& Guilfoyle, T. J. Aux/IAA proteins repress expression of reporter genes containing natural and highly active synthetic auxin response elements. Plant Cell 9, 1963-1971 (1997).

47. Friml, J. et al. Efflux-dependent auxin gradients establish the apical-basal axis of Arabidopsis. Nature 426, 147-153 (2003).

48. Clough, S. J. \& Bent, A. F. Floral dip: a simplified method for Agrobacteriummediated transformation of Arabidopsis thaliana. Plant J. 16, 735-743 (1998)

49. Beeckman, T. \& Engler, G. An easy technique for the clearing of histochemically stained plant tissue. Plant Mol. Biol. Rep. 12, 37-42 (1994).

50. Malamy, J. E. \& Benfey, P. N. Organization and cell differentiation in lateral roots of Arabidopsis thaliana. Development 124, 33-44 (1997).

51. Spitzer, M., Wildenhain, J., Rappsilber, J. \& Tyers, M. BoxPlotR: a web tool for generation of box plots. Nat. Methods 11, 121-122 (2014).

52. Karimi, M., Depicker, A. \& Hilson, P. Recombinational cloning with plant gateway vectors. Plant Physiol. 145, 1144-1154 (2007).

53. Hilson, P. et al. Versatile gene-specific sequence tags for Arabidopsis functional genomics: transcript profiling and reverse genetics applications. Genome Res. 14, 2176-2189 (2004).

54. Hellemans, J., Mortier, G., De Paepe, A., Speleman, F. \& Vandesompele, J. qBase relative quantification framework and software for management and automated analysis of real-time quantitative PCR data. Genome Biol. 8, R19 (2007).

55. Gendrel, A. V., Lippman, Z., Martienssen, R. \& Colot, V. Profiling histone modification patterns in plants using genomic tiling microarrays. Nat. Methods 2, 213-218 (2005).

56. Kaufmann, K. et al. Chromatin immunoprecipitation (ChIP) of plant transcription factors followed by sequencing (ChIP-SEQ) or hybridization to whole genome arrays (ChIP-CHIP). Nat. Protoc. 5, 457-472 (2010).

57. Jönsson, H., Heisler, M. G., Shapiro, B. E., Meyerowitz, E. M. \& Mjolsness, E. An auxin-driven polarized transport model for phyllotaxis. Proc. Natl Acad. Sci. USA 103, 1633-1638 (2006).

58. Mitchison, G. J. The dynamics of auxin transport. Proc. R. Soc. B Biol. 209, 489-511 (1980)

59. Grieneisen, V. A., Xu, J., Maree, A. F., Hogeweg, P. \& Scheres, B. Auxin transport is sufficient to generate a maximum and gradient guiding root growth. Nature 449, 1008-1013 (2007).

60. Paciorek, T. et al. Auxin inhibits endocytosis and promotes its own efflux from cells. Nature 435, 1251-1256 (2005).

61. Robert, S. et al. ABP1 mediates auxin inhibition of clathrin-dependent endocytosis in Arabidopsis. Cell 143, 111-121 (2010).

\section{Acknowledgements}

This work was supported by the National Basic Research Program of China (2015CB942900) (to Q.C. and C.L.) and (2011CB710902) (to J.L.), a European Community FP7 Marie Curie Fellowship (PIIF-GA-2012-330972) (to Q.C.), grants of the Research Foundation-Flanders (to S.V. and (FWO G.0088.12) to S.M.), of the European Research Council (project ERC-2011-StG-20101109-PSDP) (to J.F.), of the USA National Science Foundation (NSF MCB-0418891) (to E.G.), a Natural Sciences and Engineering Research Council of Canada Discovery Grant \#22R92904 (to F. S.), a Grant-in-Aid for Scientific Research on Priority Areas (19060006) from The Ministry of Education, Culture, Sports, Science and Technology, Japan (to H.F.), a FEBS long-term fellowship (to P.M.) and work in the lab of T.B. was supported by grants of the Interuniversity Attraction Poles Programme (IAP VI/33 and IUAP P7/29 'MARS') initiated by the Belgian Science Policy Office, the Research Foundation-Flanders and Ghent University Special Research Fund.

\section{Author contributions}

S.V., T.B and F.S. conceived the project. S.V., Q.C., Y.L., G.V.I., P.M., Z.X., P.M., E.L., V.V., S.K. performed general experiments. Y.L. performed yeast-one hybrid assays. S.M and K.W. performed mathematical modelling analyses. H.F., S.M., E.G., J.L. and J.F. helped analysing the data. All authors contributed to writing the manuscript.

\section{Additional information}

Supplementary Information accompanies this paper at http://www.nature.com/ naturecommunications

Competing financial interests: The authors declare no competing financial interests. 
Reprints and permission information is available online at http://npg.nature.com/ reprintsandpermissions/

How to cite this article: Chen, Q. et al. A coherent transcriptional feed-forward motif model for mediating auxin-sensitive PIN3 expression during lateral root development. Nat. Commun. 6:8821 doi: 10.1038/ncomms9821 (2015). (c) (i) This work is licensed under a Creative Commons Attribution 4.0 International License. The images or other third party material in this article are included in the article's Creative Commons license, unless indicated otherwise in the credit line; if the material is not included under the Creative Commons license, users will need to obtain permission from the license holder to reproduce the material. To view a copy of this license, visit http://creativecommons.org/licenses/by/4.0/ 